\title{
Synergistic expression of tissue factors on vascular endothelial cells induce intercellular gap formation in the presence of plasma via extrinsic coagulation cascade and PAR-1
}

Yuhki Yanase, Satoshi Morioke, Kazumasa Iwamoto, Shunsuke Takahagi, Kazue Uchida, Tomoko Kawaguchi, Kaori Ishii, Michihiro Hide

Dermatology, Hiroshima University, Japan

\section{Background}

Chronic spontaneous urticaria (CSU) is a common skin disorder characterized by daily or almost daily recurring skin edema and flare with itch. The increase of plasma histamine concentration and the effectiveness of H1-antihistamine suggests crucial role of histamine in the pathogenesis of CSU. The blood coagulation cascade and infections by microorganisms are suggested to also be involved in CSU. However, the trigger of the coagulation cascade and molecular mechanisms that link infections to wheal formation induced by mast cell degranulation are unclear. In this study, we investigated the effect of histamine and LPS on the expression of tissue factor (TF) on endothelial cells followed by activation of the coagulation pathway and intercellular gap formation.

Methods

Human Umbilical Vein Endothelial Cell (HUVEC) was used as human vascular endothelial cell. Expression of TF mRNA level in HUVECs was analyzed by RT-PCR. Surface expression level of TF on HUVECs was analyzed with flow cytometer. Activity of TF was measured by Actochrome TF activity assay. Intercellular gap formation of HUVECs was monitored with impedance sensor and fluorescent microscope.

Results

LPS and histamine synergistically induced TF expression on the surface of HUVECs, via H1 receptor and TLR-4. Moreover, synergistic expression of TF by histamine and LPS activated the extrinsic coagulation pathway resulting the activation of FXa. The reaction was effectively blocked by treatment with heparin or rivaroxaban (Xa inhibitor). Furthermore, FXa and FIIa, but not FVIIa produced by TF on HUVEC in the presence of human plasma, induced contraction of HUVECs The reactions were inhibited by vorapaxar sulfate, an antagonist of protease activated receptors (PAR)-1.

\section{Conclusions}

A small amount of histamine and LPS may induce the expression of TF by vascular endothelial cells, triggering the cascade of the extrinsic coagulation pathway followed by the increase of vascular permeability. Combined application of antagonists against histamine H1-receptor, TLRs and PAR-1, inhibitors of activated coagulation factors, such as FXa and/or agonist against adenosine receptors could be effective therapeutic targets for patients with severe and refractory CSU. 